\title{
INSUFICIENCIA VENOSA CRÓNICA Y LOS CAMBIOS ESTRUCTURALES EN LAS PAREDES DE LAS VENAS
}

(CHRONIC VENOUS INSUFFICIENCY AND STRUCTURAL CHANGES IN THE WALLS OF THE VEINS)

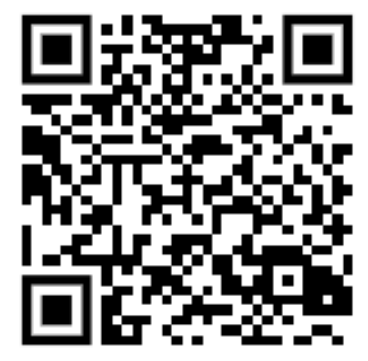

${ }^{1}$ Médico especialista en cirugía general, sub-especialista en cirugía vascular y endovascular, graduado de la Universidad de Guadalajara, médico en el Hospital Regional Universitario José María Cabral y Báez, Republica Dominicana drfaringthonreyes@gmail.com

${ }^{2}$ Médico especialista en cirugía general, graduado de la Universidad Tecnológica Republica Dominicana, médico en Hospital Regional Universitario Presidente Estrella Ureña, Republica Dominicana dr.ouelsosa@hotmail.com

\author{
${ }^{1}$ Dr. Luis Osvaldo Faringthon Reyes \\ Hospital Regional Universitario José María Cabral y Báez, República Dominicana \\ drfaringthonreyes@gmail.com \\ Dhttps://orcid.org/0000-0003-1120-3797 \\ ${ }^{2}$ Dr. Ouel Alvelis Sosa Veras \\ Hospital Regional Universitario Presidente Estrella Ureña, República Dominicana \\ dr.ouelsosa@hotmail.com \\ Dhttps://orcid.org/0000-0003-3046-6090
}

RECIBIDO

$05 / 1 / 2019$
CORREGIDO

$16 / 1 / 2019$
ACEPTADO

$22 / 1 / 2019$

\section{RESUMEN}

Objetivos: Entre todas las manifestaciones del sistema circulatorio de retorno, es quizás, la insuficiencia venosa crónica la que origina más demanda de consulta. Esta patología venosa que al principio puede cursar de forma imperceptible, logra dentro de su desarrollo generar condiciones a nivel estructural en la pared venosa que logran de forma directa activar una serie de sintomatologías que logran incapacitar de forma parcial o total a quien la padece. Es por esto que el objetivo principal de la presente revisión es identificar la mejor evidencia existente sobre la insuficiencia venosa crónica y los cambios estructurales en la pared de las venas.

Método: revisión bibliográfica, descriptiva de corte longitudinal y retrospectiva de la literatura científica controlada en base a revisiones sistemáticas.

Resultados: La evidencia obtenida confirmo que las personas que se encuentran entre los 60 y los 69 años de edad, son los de mayor prevalencia en el desarrollo de la IVC representando el $47 \%$ de la población estudiada. Seguidos por los que oscilan entre los 70 / 79 años de edad para un $26 \%$ de la muestra total de pacientes. Por otro lado en cuanto al sexo, los estudios evaluados muestran una mayor incidencia de la IVC en el sexo femenino, representando $65 \%$ de la 
población estudiada. En cuanto a los factores de riesgo para el desarrollo de la IVC los estudios evidenciaron que los no modificables son los que poseen una mayor incidencia. Se pudo apreciar entre los autores coincidencia con lo que respecta a las manifestaciones clínicas que de una forma $u$ otra sin importar que diera origen al surgimiento de la IVC, son el cuadro sintomatológico imperante en esta patología venosa. En lo que respecta a los cambios estructurales en las paredes de las venas a consecuencia de la IVC, los autores plantean que estas modificaciones se caracterizan por el engrosamiento y deformación del vaso, con aumento en su permeabilidad y pérdida de sus propiedades antitrombóticas. De igual forma describen que en el estudio histológico se revelan células endoteliales con diferentes grados de alteración. Desde aumento de sus organelos con presencia de grandes vesículas y vacuolas, cuerpos mieloides degenerativos y citoesqueleto desarrollado, hasta células que se han desprendido total 0 parcialmente de la pared venosa. La región subendotelial muestra gran infiltración colágena.

Conclusión: Dentro de los factores de riesgos predisponentes de la IVC, los estudios revisados destacan el sexo, la edad y los antecedentes familiares. De igual forma establecen que el sexo femenino es el más propenso al desarrollo de esta patología venosa y las personas que se encuentran entre los 60 y 79 años de edad. Las sintomatologías clínicas más evidentes en la IVC son la sensación de pesadez, calambres, prurito y edemas vespertinos. En lo que respecta a los cambios estructurales de la pared venosa, los autores describen un engrosamiento a nivel intimal y en la capa media, aumento de fibras colágenas, disminución de elásticas y alteración de la morfología de las células musculares lisas que pierden su orientación característica.

PALABRAS CLAVES: Insuficiencia venosa, vena safena ultrasonido.

\section{ABSTRACT}

Objectives: Among all the manifestations of the circulatory system of return, it is perhaps the chronic venous insufficiency that originates the most demand for consultation. This venous pathology that at first may occur imperceptibly, achieves within its development to generate conditions at the structural level in the venous wall that directly activate a series of symptoms that partially or totally incapacitate the sufferer. This is why the main objective of this review is to identify the best existing evidence on chronic venous insufficiency and structural changes in the vein wall.

Method: bibliographical review, descriptive of longitudinal section and retrospective of the controlled scientific literature based on systematic reviews.

Results: The evidence obtained confirmed that people between 60 and 
69 years of age are the most prevalent in the development of CVI, representing $47 \%$ of the population studied. Followed by those who range between $70 / 79$ years of age for $26 \%$ of the total sample of patients. On the other hand, in terms of sex, the studies evaluated show a higher incidence of $\mathrm{CVI}$ in females, representing $65 \%$ of the population studied. Regarding the risk factors for the development of the IVC, the studies showed that the non-modifiable ones have the highest incidence. It was possible to see among the authors coincidence with regard to the clinical manifestations that in one way or another regardless of which gave rise to the emergence of CVI, are the symptomatological picture prevailing in this venous pathology. Regarding the structural changes in the walls of the veins as a consequence of the IVC, the authors suggest that these modifications are characterized by the thickening and deformation of the vessel, with an increase in its permeability and loss of its antithrombotic properties. They also describe that in the histological study, endothelial cells with different degrees of alteration are revealed. From the increase of its organelles with the presence of large vesicles and vacuoles, degenerative myeloid bodies and developed cytoskeleton, to cells that have completely or partially detached from the venous wall. The subendothelial region shows great collagen infiltration.

Conclusion: Among the risk factors predisposing to CVI, the studies reviewed include gender, age and family history. They also establish that the female sex is the most prone to the development of this venous pathology and people who are between 60 and 79 years of age. The most evident clinical symptomatology in the IVC is the sensation of heaviness, cramps, pruritus and evening edemas. With regard to the structural changes of the venous wall, the authors describe a thickening at the intimal level and in the middle layer, increase in collagen fibers, decrease in elastics and alteration of the morphology of smooth muscle cells that lose their characteristic orientation.

KEYWORDS: Venous insufficiency, saphenous vein, ultrasound.

\section{DESARROLLO}

La Insuficiencia Venosa Crónica (IVC), es una alteración funcional del retorno venoso, debido a una insuficiencia o incompetencia valvular venosa con o sin obstrucción venosa. Son muchos los síntomas que pueden aparecer en los primeros estadios de esta enfermedad.
Los principales para la mayoría de los pacientes son dolor, pesadez, calambres nocturnos, sensación de tumefacción y prurito en los miembros inferiores. En los primeros estadios de la enfermedad los síntomas pueden ser la única manifestación de esta, que abarca una amplia variedad de tipos y de intensidad, que pueden ser leves o graves. Es, por tanto, importante mejorar los síntomas y 
comenzar el tratamiento desde sus primeras manifestaciones clínicas $(1,2)$. La circulación sanguínea a través del sistema venoso se debe al sistema valvular, pero también a la bomba músculo esquelético. Durante la marcha, la contracción muscular exprime la sangre desde el sistema venoso superficial (SVS) a través de las venas comunicantes al sistema venoso profundo (SVP), y por éste a la vena femoral común e iliaca.

Sin embargo, en el caso de insuficiencia valvular, parte de esta sangre retorna al sistema superficial que de esta manera ve sobrecargado su volumen sanguíneo en una cantidad extra. Este volumen hemático no tiene un efecto hemodinámico marcado sobre la totalidad del sistema circulatorio, pero sí lo tiene sobre la circulación regional del miembro afectado (3-5).

\section{EPIDEMIOLOGÍA}

Se calcula que 25 a $50 \%$ de la población adulta mundial puede estar afectada por este síndrome clínico. La incidencia y prevalencia de las varices se han estudiado en una serie de protocolos transversales.

En 1973, en el estudio de salud de comunidad en Tecumseh, Michigan, se calculó que alrededor de 40 millones de personas (26 millones de mujeres) en Estados Unidos se vieron afectados (6). En 1994, una revisión por parte de Callam, encontró que la mitad de la población adulta tiene los estigmas de menor importancia de la enfermedad venosa $(50-55 \%$ las mujeres, $40-50 \%$ los hombres) y menos de la mitad muestra venas varicosas visibles $(20-25 \%$ las mujeres, 10-15\% los hombres), en 2004 estos hallazgos se observaron también en un estudio transversal francés que encontró la razón de posibilidades al año para las venas varicosas de 1.04 para las mujeres y 1.05 para los hombres. La edad y el género han sido los únicos factores de riesgo identificado de forma consistente para las venas varicosas (79).

\section{ETIOLOGÍA}

La causa principal es la insuficiencia valvular de las venas superficiales, con mayor frecuencia en la unión safenofemoral.

De forma secundaria, es posible también la trombosis venosa profunda (TVP) que conduce a la obstrucción crónica venosa profunda o la insuficiencia valvular. Las secuelas a largo plazo de esta anomalía se conocen como síndrome postrombótico y también deben incluirse los problemas asociados a catéter por TVP (10).

Otras causas son la pared venosa inducida por el embarazo y la progesterona y la debilidad de la válvula agravada por el volumen de sangre circulante; la ampliación de útero agrandado, lo cual comprime la vena cava inferior y el retorno venoso desde las extremidades inferiores; los traumatismos; y las alteraciones congénitas, incluidas todas las malformaciones venosas (p. ej., KlippelTrenaunay y variantes, y avalvulia) $(11,12)$. 


\section{FISIOPATOLOGÍA}

La insuficiencia venosa es consecuencia de la afección de una o varias de las venas de las piernas. En condiciones normales, la circulación venosa de las piernas está constituida por el sistema venoso profundo, las venas superficiales y las venas comunicantes. La dirección del flujo se proyecta desde el sistema superficial hasta el sistema profundo. El retorno venoso tiene lugar por un bajo flujo impulsado por las arterias (llamado vis a tergo) e influyen también la presencia de válvulas unidireccionales que impiden el regreso del flujo. Otros factores que contribuyen al retorno venoso son la presión negativa de la cavidad torácica, sobre todo cuando se realiza la inspiración y la contracción muscular, la cual actúa como bomba e impulsa el flujo sanguíneo con la ayuda de las válvulas (13).

El aumento de flujo dilata las venas y las válvulas se vuelven insuficientes. Además de la trombosis, las venas pueden dilatarse por algunos factores, entre ellos el embarazo (por las concentraciones elevadas de progesterona), sexo femenino, edad, alta estatura, obesidad, factores genéticos y largos periodos de pie o sentado (14).

\section{CUADRO CLÍNICO}

Los síntomas son de dos tipos: aquellos pacientes que se quejan de sensación de pesadez en las piernas y edema pretibial de toda la extremidad, y los individuos que sólo refieren venas dilatadas, a las cuales se las denomina varices, con tortuosidades y estéticamente desagradables. Cuando el problema persiste sin tratamiento adecuado, las varices pueden dar lugar a que la elevación de la presión hidrostática de las venas produzca pequeñas hemorragias de tipo petequial y que este tipo de sangrado crónico cause con posterioridad una tinción color ocre de la piel de las extremidades inferiores. Cuando la insuficiencia venosa se acompaña de trombosis profunda, se presenta en la forma de edema importante de la extremidad, dolor y cambios en la coloración. Otros síntomas que pueden presentarse son cansancio, prurito, dolor urente y dolor punzante (15).

\section{MODIFICACIONES ESTRUCTURALES $Y$ FUNCIONALES EN LA IVC}

\section{Alteraciones en la pared venosa}

El estudio ultraestructural de la pared venosa insuficiente muestra cómo sus células componentes tratan de compensar las necesidades de la pared ante la hipertensión-hipoxia mantenida. La observación histológica deja ver células endoteliales con diferentes grados de alteración.

Desde aumento de sus organelos con presencia de grandes vesículas y vacuolas, cuerpos mieloides degenerativos y citoesqueleto desarrollado, hasta células que se han desprendido total o parcialmente de la pared venosa. La región subendotelial muestra gran infiltración colágena $(16,17)$.

Una muestra de la disfunción del tejido endotelial en la vena varicosa está dada 
por la reducción observada en la contractilidad dependiente del factor constrictor derivado del endotelio (EDCF) después de estímulos con noradrenalina. Desde las primeras etapas de la IVC se producen cambios funcionales en las SMC, las cuales proliferan localmente favoreciendo el engrosamiento de la pared. Estas SMC participan en la producción de grandes cantidades de tejido conectivo y de enzimas lisosomales degenerativas. Esto es posible pues a diferencia del vaso sano, donde coexisten de acuerdo con las necesidades del sector venoso dos fenotipos de SMC, el de tipo metabólico y el contráctil. En la vena varicosa este equilibrio se encuentra desplazado hacia el fenotipo metabólico en detrimento de los filamentos contráctiles de su estructura (18).

La alteración estructural más importante de las venas varicosas parece corresponder a las modificaciones en los componentes del tejido conectivo. Aunque la literatura refiere incongruencias en cuanto a resultados, la mayoría de los autores afirman haber encontrado disminución del contenido de elastina y aumento del contenido de proteoglicanos y colágeno; con disminución del colágeno tipo fibrilar, abundando el colágeno de tipo membranoso, en comparación con venas normales (19).

El tejido conectivo no sólo se modifica en la proporción de sus componentes, sino que se aprecian abundantes fibras colágenas y elásticas displásicas.

El colágeno se dispone desorganizadamente, fragmentado, distorsionado, formando bucles sin dirección definida y la fibra elástica aparece frecuentemente fragmentada, tortuosa, más gruesa o ausente, lo cual es evidente en los segmentos venosos dilatados. Según un estudio reciente se pudo demostrar que son varias las características morfológicas de la vena safena superficial que correlacionan con la severidad de la enfermedad venosa (20,21).

Recientemente se ha demostrado que la neuropatía sensorial es una característica de la IVC y que su distribución coincide con la de los cambios tróficos. Al no ser apreciada por el paciente probablemente esta neuropatía contribuya al deterioro de traumas menores al que se encuentra sujeto (22).

\section{Interacciones de las células} sanguíneas con el endotelio venoso

Las células endoteliales gracias a su posición de barrera limítrofe entre la sangre y el vaso están capacitadas para recibir y responder señales de su medio ambiente que constituyen actividades claves para la vida. Se interconectan con células de la sangre y el vaso a través de comunicaciones auto y paracrinas que les permiten controlar el metabolismo de la pared respondiendo rápidamente a necesidades locales.

Los leucocitos, especialmente los granulocitos, por ser células altamente reactivas mantienen intensas interacciones con el endotelio, incluso en condiciones fisiológicas. La elevada reactividad del leucocito determina su participación en la fisiopatología de las enfermedades vasculares, como es el caso de la IVC (23). 
Se ha demostrado que las CE activadas por hipoxia sintetizan y liberan factor activante plaquetario (PAF), un mediador inflamatorio muy fuerte, cuya acción promueve la adhesión firme del leucocito, su activación y migración posterior al espacio subendotelial. Las moléculas de adhesión que intervienen en esta unión firme y en la transmigración del leucocito son integrinas leucocitarias. En neutrófilos y monocitos esta interacción está mediada por integrinas de la familia de la B2: LFA1; mac-1 y la p150, 95. Adicionalmente en los monocitos también interviene la integrina VLA-4 de la familia B1 y otras integrinas de la B3. Como contrarreceptor en el endotelio ya activado moléculas de adhesión de la superfamilia de las inmunoglobulinas tales como ICAM-1 y VCAM-1 (24-26). Los leucocitos así activados segregan a su vez mediadores inflamatorios, entre ellos: PAF leucotrienos y citoquinas $y$ una descarga citotóxica altamente degenerativa de radicales libres de oxígeno y proteasas que degeneran el vaso y provocan la extravasación de proteínas plasmáticas dando paso a la formación de edemas. Esta actividad desplegada por los leucocitos forma parte de su función en el sistema inespecífico de defensa, atrayendo más leucocitos al lugar y preparando las condiciones para facilitar su migración al tejido. Específicamente por estímulo del PAF, liberado tanto por el endotelio hipóxico como por los leucocitos, puede ocurrir, como su nombre indica, la activación espontánea de plaquetas. En estado de activación las plaquetas a su vez segregan factores inflamatorios como IL-114 y PAF, y agentes procoagulantes, proagregantes, mitógenos e inductores de permeabilidad del vaso, favoreciéndose la formación de trombina $(27,28)$.

En lugares de estasis, la velocidad del flujo sanguíneo disminuida permite la acumulación de células de la sangre y la concentración de factores de la coagulación activados, potenciándose la formación de trombos en ciertos puntos del árbol venoso, con predilección en las bolsas valvulares; en tanto se hace difícil la llegada de inhibidores del proceso. Particularmente en las venas varicosas, al estar disminuida la síntesis de PGI2 no existe una adecuada protección contra la agregación plaquetaria y su descarga trombogénica (29).

\section{Trastornos en la microcirculación}

Todas estas reacciones intercelulares a las que se ha hecho referencia cobran mayor importancia en la microcirculación donde hay un contacto más estrecho entre la sangre y la pared del vaso. Normalmente las células circulantes, es especial los leucocitos, durante su paso por los capilares adoptan formas alargadas y estrechas para no contactar con el endotelio. Al ser los leucocitos más rígidos que los eritrocitos demoran más que estos en deformarse y viajan más lentamente afectando la cinética del flujo. Los eritrocitos, o sus agregados en dependencia del grosor del vaso, tienden a adelantarse a los leucocitos y desplazarlos hacia la periferia, facilitándose de esta forma su contacto con el endotelio y su adhesión en sitios donde exista algún daño $(30,31)$.

La situación se agrava cuando estamos en presencia de hipertensión venosa. Característicamente en estas condiciones se produce una alteración en la relación de presiones capilar- 
vénula poscapilar, que provoca disminución de la velocidad del flujo. El endotelio al sufrir hipoxia se altera, segrega PAF y por relajación viscoelástica presenta proyecciones hacia la luz del vaso. El contacto del leucocito con el endotelio, su adhesión y activación se ven favorecidos por la expresión incrementada de moléculas de adhesión celular en leucocitos y endotelio. Además con la reducción del flujo a nivel de la microcirculación hay menos probabilidad de arrastre mecánico de los leucocitos por la corriente sanguínea y estas células quedan atrapadas (32).

Se ha demostrado que esto ocurre en capilares de miembros inferiores de pacientes con IVC. De acuerdo con un análisis realizado en sangre venosa del pie en estos pacientes aproximadamente un $30 \%$ de los leucocitos no regresa a la circulación después de mantener sus extremidades una hora en posición declive. En comparación, sólo se demostró una reducción de un $5 \%$ en los leucocitos circulantes de personas sanas en igualdad de condiciones (33).

El atrapamiento de leucocitos en los capilares de los miembros inferiores causa, entre otras implicaciones, lesión vascular, aparición del fenómeno de noreflujo y produce alteración inmunológica pues los leucocitos secuestrados tienen pocas posibilidades de contactar con microorganismos (34).

Las alteraciones descritas comprometen tanto a los capilares que deben nutrir al tejido como a las vénulas poscapilares que deben transportar los productos de desecho de su metabolismo. Cuando un gran número de vasos capilares está alterado el defecto nutritivo provoca lesiones tisulares características de la IVC, la dermolipoesclerosis y la úlcera (35-37).

Se concluye que en la pared de las venas insuficientes predominan las células musculares lisas de fenotipo metabólico, las que producen grandes cantidades de matriz extracelular y enzimas degenerativas, modificándose el contenido y calidad de los componentes del tejido conectivo. El vaso se engrosa y deforma, desarrollando una actividad contráctil baja. Las células endoteliales activadas por la hipertensión mantenida hacen que se favorezcan las interacciones entre las células sanguíneas y la pared del vaso, que aumenta su permeabilidad con pérdida de sus propiedades antitrombóticas, lo que se asocia a la formación de edemas y trombos. Los trastornos hemodinámicos venosos provocan hipertensión capilar, agudizándose las interacciones celulares entre la sangre y el vaso a este nivel. Los cambios en la microcirculación conllevan a la aparición de las lesiones características de la insuficiencia venosa crónica (38).

\section{DIAGNÓSTICO DE LA IVC}

El diagnóstico debe realizarse primero por métodos clínicos. Hay que recordar que la anomalía se presenta en los miembros inferiores, el sistema venoso profundo, el sistema comunicante y las venas superficiales. Estas últimas están constituidas por el sistema de la safena interna y el sistema de la safena externa. La safena interna recorre la pierna por la cara interna, asciende por el muslo hasta desembocar en la femoral mediante el 
cayado de la safena (existe una válvula llamada osteal). La safena externa es más corta, se extiende por la pierna en su borde externo, se proyecta hacia el hueco poplíteo y se anastomosa con la vena poplítea (39).

\section{TRATAMIENTO PARA LA IVC}

El tratamiento de las varices depende de los síntomas, la cronicidad de la enfermedad y posibles complicaciones. Como tratamiento no farmacológico es necesario realizar ejercicio para activar la bomba muscular, tener las extremidades elevadas cuando se encuentre el paciente en descanso y es necesario perder peso.

Existen otros métodos para esclerosar varices, entre ellos la esclerosis con el uso de láser y el empleo de un catéter de radiofrecuencia, el cual tiene funciones muy similares a las del láser. Se requiere permeabilidad del sistema venoso profundo y que las varices no tengan tortuosidad excesiva. Estos dos métodos se realizan con anestesia local y sedación y se han informado buenos resultados con una duración de cinco a siete años $(40,41)$.

\section{RESULTADOS DE LA REVISIÓN}

Mediante la presente revisión se evaluó la insuficiencia venosa crónica (IVC) y los cambios estructurales en las paredes de las venas (CEPV). Considerando una muestra total evaluada de 796 pacientes, se obtuvieron los siguientes resultados (42-44):

\section{Cambios estructurales en las paredes de las venas}

Dentro de los resultados arrojados por los estudios incluidos en la presente revisión con relación a los cambios estructurales en las paredes de las venas a consecuencia de la insuficiencia venosa crónica (IVC), se puedo constatar:

- La aparición de las varices que son dilataciones tortuosas de las venas superficiales, típicamente de miembros inferiores, aparecen como consecuencia de la insuficiencia valvular venosa y la incapacidad de mantener un flujo sanguíneo eficaz hacia el corazón.

- Los mismos identificaron factores predisponentes para la aparición de varices, tales como el sexo, la gestación, el peso, la altura, la raza, la dieta y hábitos intestinales, el trabajo, la postura, haber sufrido una trombosis venosa profunda previamente y determinados marcadores genéticos. Aun así, el proceso patogénico por el cual venas sanas degeneran en varicosas aún no está claro.

- Dentro de los mediadores moleculares implicados, que se describen los más relevantes son el factor de crecimiento transformante beta (TGF- $\beta$ ), otros factores de crecimiento (como PDGF, VEGF), metaloproteinasas (MMP-2), disminución de la óxido nítrico sintasa y aumento de radicales libres en las venas varicosas, entre otros. Todo ello conlleva un aumento en la actividad apoptótica a través de distintas vías de señalización intracelular, sobre todo a nivel de la 
capa media y aumento de la fibrosis en la pared de la vena.

- La distensibilidad de la pared de la vena está determinada por la proporción de colágeno, elastina y células musculares lisas que la forman. Sin embargo, existen evidencias contradictorias acerca de la concentración de tejido conectivo y tejido muscular liso patológico en las venas varicosas. Algunos autores han demostrado una menor concentración de fibras colágenas y elásticas en venas varicosas, mientras que otros han encontrado una concentración mayor de colágeno y similar de fibras elásticas. También se ha hablado de una menor concentración de fibras elásticas y mayor de colágenas.

\section{Resultados histológico en las venas varicosas.}

Los autores explican que en las venas varicosas mediante el estudio histológico puede apreciarse una hipertrofia intimal, debido principalmente a la infiltración de tejido colágeno en dicha capa y la posible emigración de células musculares lisas, que se observan a dicho nivel en algunos cortes. Además, la superficie intimal es más irregular que en la vena sana, con discontinuidad del endotelio en algunos tramos. El grosor de la capa media es también significativamente mayor. Al observar los cortes a gran aumento puede observarse que las células musculares lisas pierden su morfología habitual en huso y finas, mostrándose hipertróficas con una forma más.

\section{DISCUSIÓN DE LA REVISIÓN}

La insuficiencia venosa crónica (IVC) es una condición patológica del sistema venoso que se caracteriza por la incapacidad funcional adecuada del retorno sanguíneo debido a anormalidades de la pared venosa y valvular que lleva a una obstrucción o reflujo sanguíneo en las venas. La Unión Internacional de Flebología define la IVC como los cambios producidos en las extremidades inferiores resultado de la hipertensión venosa prolongada.La evidencia obtenida confirmo que las personas que se encuentran entre los 60 y los 69 años de edad, son los de mayor prevalencia en el desarrollo de la IVC representando el $47 \%$ de la población estudiada. Seguidos por los que oscilan entre los 70 / 79 años de edad para un $26 \%$ de la muestra total de pacientes (TABLA 1.1 / GRAFICO 1.1).

Apoyando esto lo descrito en los estudios $81,83,85$ que especifican que los pacientes que se encuentran entre los 60 y los 79 años de edad, son los más vulnerables al desarrollo de la IVC.

Por otro lado en cuanto al sexo, los estudios evaluados muestran una mayor incidencia de la IVC en el sexo femenino, representando $65 \%$ de la población estudiada. Corroborando esto lo descrito por varios autores donde aseguran que la IVC es más frecuente en mujeres que en los hombres. Siendo de igual forma uno de los factores de riesgo no modificables de mayor prevalencia (TABLA 1.2 / GRAFICO 1.2). 
GRÁFICO 1.1. Población estudiada según grupos etéreos.

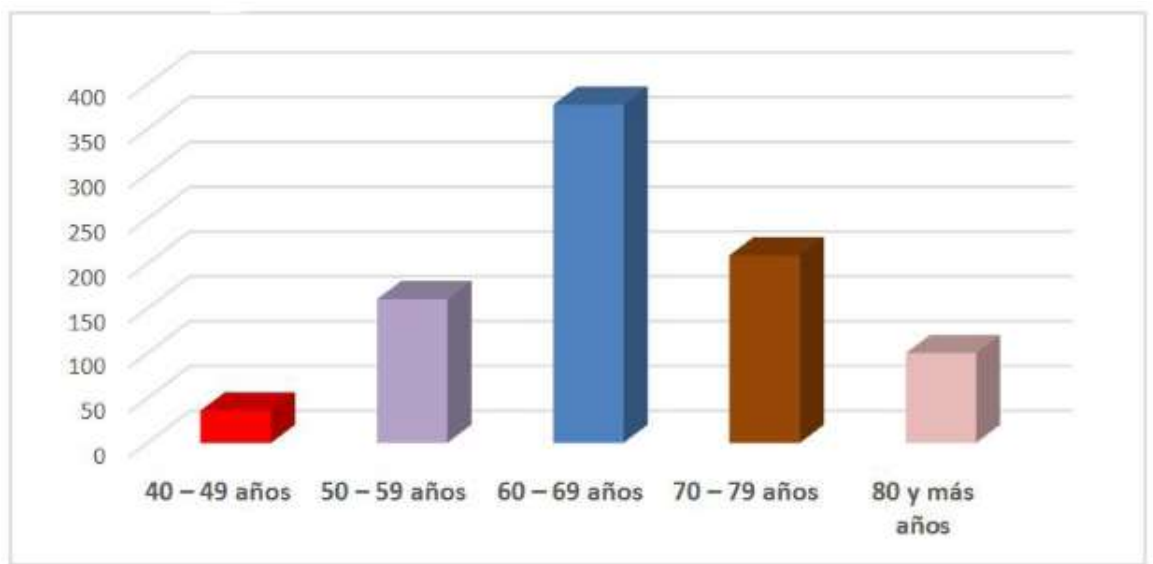

Fuente: estudios incluidos.

GRÁFICO 1.2. Población estudiada según sexo

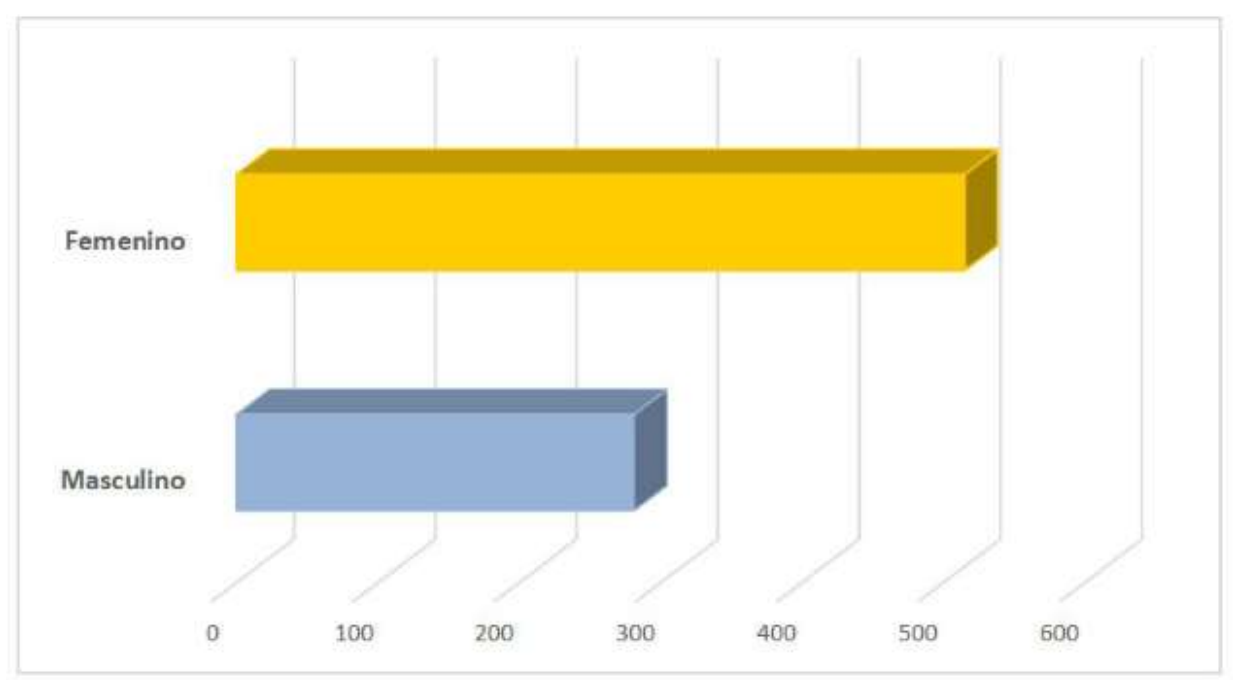

Fuente: estudios incluidos.

TABLA 1.1. Distribución IVC según grupos etéreos.

\begin{tabular}{|c|c|c|}
\hline Edad & Frecuencia & Porcentaje \\
\hline 40-49 años & 36 & $5 \%$ \\
\hline 50-59 años & 160 & $20 \%$ \\
\hline 60-69 años & 377 & $47 \%$ \\
\hline 70-79 años & 209 & $26 \%$ \\
\hline 80 y más años & 14 & $2 \%$ \\
\hline Total & 796 & $100 \%$ \\
\hline \multicolumn{3}{|c|}{ Fuente: estudios incluidos. } \\
\hline
\end{tabular}

TABLA 1.2. Distribución IVC según sexo.

\begin{tabular}{|c|c|c|}
\hline & Frecuencia & Porcentaje \\
\hline Masculino & 281 & $35 \%$ \\
\hline Femenino & 515 & $65 \%$ \\
\hline TOTAL & 796 & $100 \%$ \\
\hline Fuente: estudios incluidos. \\
\hline
\end{tabular}

En cuanto a los factores de riesgo para el desarrollo de la IVC los estudios evidenciaron que los no modificables son los que poseen una mayor incidencia. 


\section{GRÁFICO 1.3 Población estudiada según factor de riesgo}

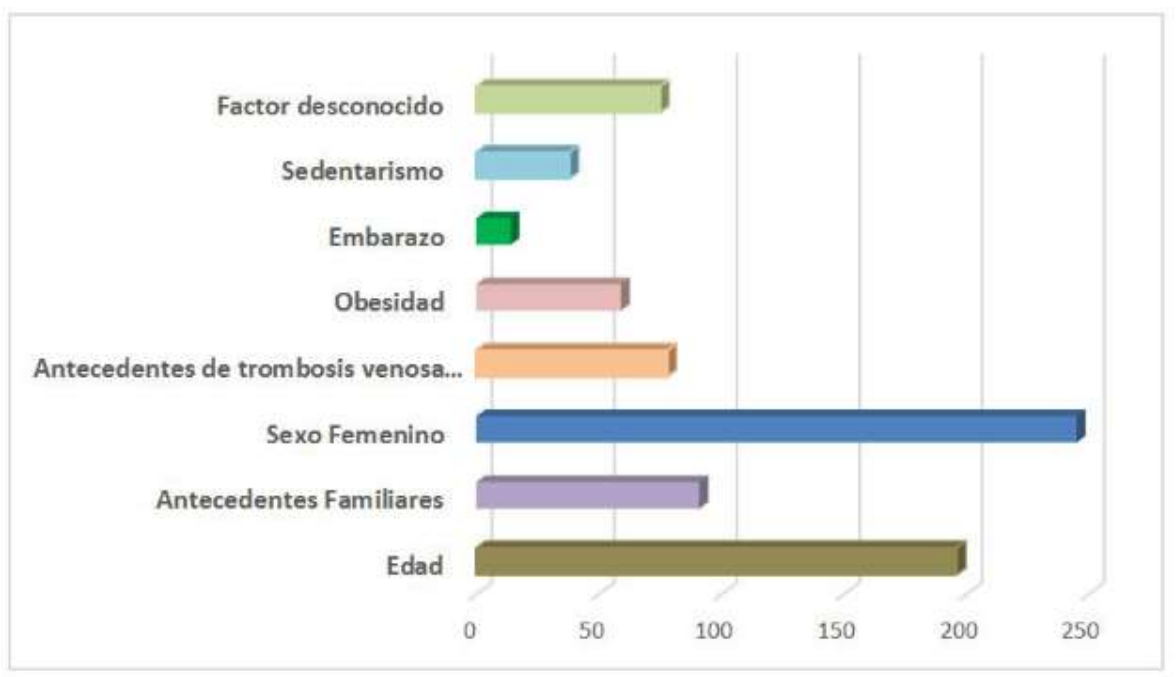

Fuente: estudios incluidos.

\section{GRÁFICO 1.4 Población estudiada según manifestaciones clínicas}

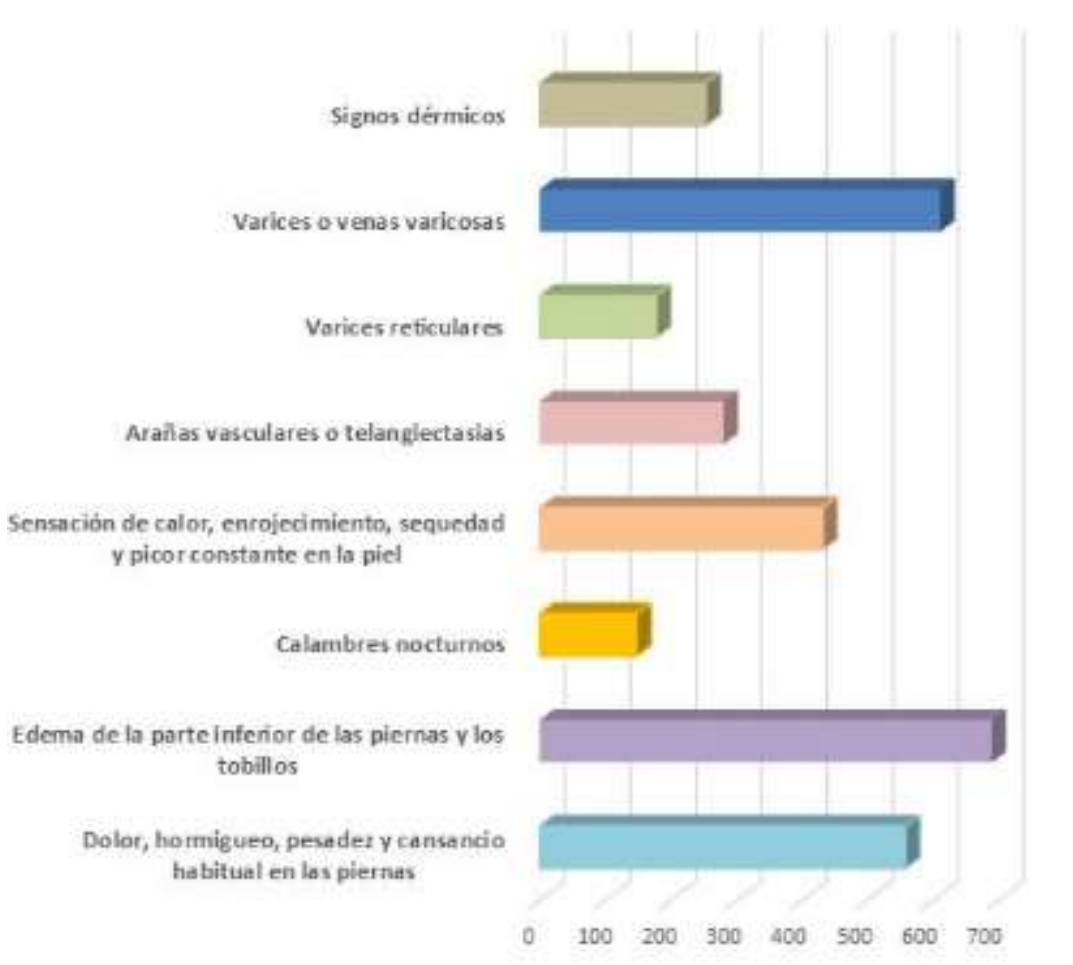

Fuente: estudios incluidos. 
Sin embargo otros autores sostienen que algunos de los factores modificables y sobre todo aquellos que tienen que ver con el estilo de vida de la persona inciden de forma directa en el surgimiento de la IVC (TABLA 1.3 / GRÁFICO 1.3).

\begin{tabular}{|c|c|c|}
\hline \multicolumn{3}{|c|}{$\begin{array}{l}\text { TABLA 1.3. Distribución IVC según } \\
\text { factor de riesgo. }\end{array}$} \\
\hline $\begin{array}{l}\text { Factores de } \\
\text { riesgo }\end{array}$ & Frecuencia & Porcentaje \\
\hline $\begin{array}{c}\text { NO } \\
\text { MODIFICABLES }\end{array}$ & & \\
\hline Edad & 196 & $25 \%$ \\
\hline $\begin{array}{l}\text { Antecedentes } \\
\text { familiares }\end{array}$ & 91 & $11 \%$ \\
\hline Sexo femenino & 245 & $31 \%$ \\
\hline MODIFICABLES & & \\
\hline $\begin{array}{l}\text { Antecedente de } \\
\text { trombosis venosa } \\
\text { profunda en las } \\
\text { piernas }\end{array}$ & 78 & $10 \%$ \\
\hline Obesidad & 59 & $7 \%$ \\
\hline Embarazo & 14 & $2 \%$ \\
\hline Sedentarismo & 38 & $5 \%$ \\
\hline Factor desconocido & 75 & $9 \%$ \\
\hline TOTAL & 796 & $100 \%$ \\
\hline
\end{tabular}

Se pudo apreciar entre los autores coincidencia con lo que respecta a las manifestaciones clínicas que de una forma $u$ otra sin importar que diera origen al surgimiento de la IVC, son el cuadro sintomatológico imperante en esta patología venosa

\section{GRAFICO 1.4).}

(TABLA1.4 /

\begin{tabular}{|l|l|l|}
\hline \multicolumn{3}{|c|}{$\begin{array}{l}\text { TABLA 1.4. Manifestaciones clínicas } \\
\text { presentes en la población estudiada en } \\
\text { base a la evaluación de la muestra total. }\end{array}$} \\
\hline $\begin{array}{c}\text { Manifestaciones } \\
\text { Clínicas }\end{array}$ & $\begin{array}{c}\text { Cantidad } \\
\text { de } \\
\text { pacientes }\end{array}$ & Porcentaje \\
\hline $\begin{array}{l}\text { Dolor, hormigueo, } \\
\text { pesadez y cansancio } \\
\text { habitual } \text { en las } \\
\text { piernas }\end{array}$ & 558 & $70 \%$ \\
\hline $\begin{array}{l}\text { Edema de la parte } \\
\text { inferior de las } \\
\text { piernas y los tobillos }\end{array}$ & 690 & $86 \%$ \\
\hline $\begin{array}{l}\text { Calambres } \\
\text { nocturnos }\end{array}$ & 148 & $18 \%$ \\
\hline $\begin{array}{l}\text { Sensacion de calor, } \\
\text { enrojecimiento, } \\
\text { sequedad y prurito } \\
\text { constante en la piel }\end{array}$ & 432 & $54 \%$ \\
\hline $\begin{array}{l}\text { Arañas vasculares o } \\
\text { telagiectasias }\end{array}$ & 281 & $35 \%$ \\
\hline Varices reticulares & 178 & $22 \%$ \\
\hline $\begin{array}{l}\text { Varices o venas } \\
\text { varicosas }\end{array}$ & 611 & $77 \%$ \\
\hline Signo dérmicos & 254 & $32 \%$ \\
\hline Fuente: estudios incluidos. & \\
\hline
\end{tabular}

En lo que respecta a los cambios estructurales en las paredes de las venas a consecuencia de la IVC, los autores plantean que estas modificaciones se caracterizan por el engrosamiento y deformación del vaso, con aumento en su permeabilidad y pérdida de sus propiedades antitrombóticas. 
De igual forma describen que en el estudio histológico se revelan células endoteliales con diferentes grados de alteración. Desde aumento de sus organelos con presencia de grandes vesículas y vacuolas, cuerpos mieloides degenerativos y citoesqueleto desarrollado, hasta células que se han desprendido total o parcialmente de la pared venosa. La región subendotelial muestra gran infiltración colágena.

La microbiota a nivel del cuerpo humano tiene un papel importante que aún esta en investigación. En el caso del sistema genital femenino predomina la presencia de Lactobacilos spp., que mediante distintos mecanismos producen un ambiente de defensa ante patógenos oportunistas. Existen factores, tanto modificables como no modificables. Los últimos, es necesario tomarlos en cuenta para mejorar el ambiente vaginal.

Debido a esto es importante dar a conocer los variables modificables, lo cual podría prevenir complicaciones en la salud de mujeres en edad reproductiva y en estado de embarazo.

\section{REFERENCIAS}

1. Gruss J, Hiemer W. Results of femoropopliteal and femorotibial greater saphenous vein in situ bypass. Life table analysis. International Angiology. 1992;11(2):94-105.

2. Husfeldt K. Venous replacement with Gore-tex prosthesis: experimental and first clinical results. Pelvic and abdominal veins: Progress in diagnosis and therapy. Excerpta Medica; Amsterdam. 1981, 249-258.

3. ljima $\mathrm{H}$, Kodama $\mathrm{M}$, Hori $\mathrm{M}$. Temporary arteriovenous fistula for venous reconstruction using synthetic graft: a clinical and experimental investigation. The Journal of Cardiovascular Surgery. 1985;26(2):131-136.

4. Plate G, Einarsson E, Eklöf B, Jensen R, Ohlin P. Iliac vein obstruction associated with acute iliofemoral venous thrombosis. Results of early reconstruction using polytetrafluoroethylene grafts. Acta Chir Scand. 1985;151(7):607-611.

5. Okadome K. Venous Reconstruction for lliofemoral Venous Occlusion Facilitated by Temporary

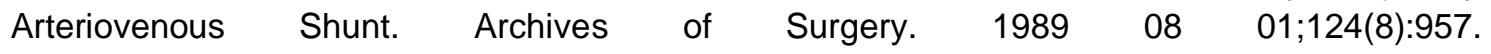
https://doi.org/10.1001/archsurg. 1989.01410080093015

6. Gloviczki P, Pairolero PC, Toomey BJ, Bower TC, Rooke TW, Stanson AW, Hallett JW, Cherry KJ. Reconstruction of large veins for nonmalignant venous occlusive disease. Journal of Vascular Surgery. 1992 Nov;16(5):750-761. https://doi.org/10.1016/0741-5214(92)90230-6 
INSUFICIENCIA VENOSA CRÓNICA Y LOS CAMBIOS ESTRUCTURALES EN LAS PAREDES DE LAS

VENAS - Dr. Luis Osvaldo Faringthon Reyes / Dr. Ouel Alvelis Sosa Veras

doi : https://doi.org/10.31434/rms.v4i2.172

7. Alimi $Y$, DiMauro $P$, Fabre D, Juhan C. lliac vein reconstructions to treat acute and chronic venous occlusive disease. Journal of Vascular Surgery. 1997 04;25(4):673-681. https://doi.org/10.1016/s0741-5214(97)70294-5

8. Jost CJ, Gloviczki P, Cherry KJ, McKusick MA, Harmsen WS, Jenkins GD, Bower TC. Surgical reconstruction of iliofemoral veins and the inferior vena cava for nonmalignant occlusive disease. Journal of Vascular Surgery. $2001 \quad$ 02;33(2):320-328. https://doi.org/10.1067/mva.2001.112805

9. Neglén P, Hollis KC, Olivier J, Raju S. Stenting of the venous outflow in chronic venous disease: Long-term stent-related outcome, clinical, and hemodynamic result. Journal of Vascular Surgery. 2007 Nov;46(5):979-990.e1. https://doi.org/10.1016/j.jvs.2007.06.046

10. Hartung O, Loundou A, Barthelemy P, Arnoux D, Boufi M, Alimi Y. Endovascular Management of Chronic Disabling Ilio-caval Obstructive Lesions: Long-Term Results. European Journal of $\begin{array}{llll}\text { Vascular and } \quad \text { Endovascular } & \text { Surgery. } & & \end{array}$ https://doi.org/10.1016/j.ejvs.2009.03.004

11. Ye K, Lu X, Li W, Huang Y, Huang X, Lu M, Jiang M. Long-Term Outcomes of Stent Placement for Symptomatic Nonthrombotic lliac Vein Compression Lesions in Chronic Venous Disease. Journal of Vascular and Interventional Radiology. 2012 04;23(4):497-502. https://doi.org/10.1016/j.jvir.2011.12.021

12. Rosales A, Sandbæk G, Jørgensen J. Stenting for Chronic Post-thrombotic Vena Cava and Iliofemoral Venous Occlusions: Mid-term Patency and Clinical Outcome. European Journal of Vascular and Endovascular $\quad$ Surgery. $2010 \quad$ 08;40(2):234-240. https://doi.org/10.1016/..ejvs.2010.04.016

13. Kölbel $T$, Lindh $M$, Åkesson $M$, Wassèlius J, Gottsäter A, Ivancev K. Chronic lliac Vein Occlusion:Midterm Results of Endovascular Recanalization. Journal of Endovascular Therapy. 2009 08;16(4):483-491. https://doi.org/10.1583/09-2719.1

14. Allegra C, Antignani P, Bergan JJ, Carpentier PH, Coleridge-Smith $\mathrm{P}$, Cornu-Thénard A, Eklof B, Partsch H, Rabe E, Uhl J, Widmer M. The "C" of CEAP: Suggested definitions and refinements: An international union of phlebology conference of experts. Journal of Vascular Surgery. 2003 01;37(1):129-131. https://doi.org/10.1067/mva.2003.47

15. Gesto-Castromil R, García J. Encuesta epidemiológica realizada en España sobre la prevalencia asistencial de la insuficiencia venosa crónica en atención primaria. Estudio DETECT-IVC. Angiología. 2001 01;53(4):249-260. https://doi.org/10.1016/s0003$\underline{3170(01) 74698-6}$

16. Browse N, Burnand K, Irvine A, Wilson N. Varicose veins: Pathology. Oxford University Press; 1999. 
INSUFICIENCIA VENOSA CRÓNICA Y LOS CAMBIOS ESTRUCTURALES EN LAS PAREDES DE LAS

VENAS - Dr. Luis Osvaldo Faringthon Reyes / Dr. Ouel Alvelis Sosa Veras

doi : https://doi.org/10.31434/rms.v4i2.172

17. Cornu-Thenard A, Boivin P, Baud J, De Vincenzi I, Carpentier P. Importance of the familial factor in varicose disease. Clinical study of 134 families. Journal of Dermatology \& Dermatologic Surgery. 1994;20(5):318-326.

18. Raffetto JD, Khalil RA. Mechanisms of varicose vein formation: valve dysfunction and wall dilation. Phlebology: The Journal of Venous Disease. 2008 04;23(2):85-98. https://doi.org/10.1258/phleb.2007.007027

19. Atta HM. Varicose Veins: Role of Mechanotransduction of Venous Hypertension. International Journal of Vascular Medicine. 2012;2012:1-13. https://doi.org/10.1155/2012/538627

20. Haviarova Z, Janegova A, Janega P, Durdik S, Kovac P, Stvrtinova V, Mraz P. Nitric oxide synthases in varicose vein wall. Bratislava Medical Journal. 2011;112(1):18-23.

21. Filis $K$, Kavantzas N, Isopoulos $T$, Antonakis $P$, Sigalas $P$, Vavouranakis $E$, Sigala F. Increased Vein Wall Apoptosis in Varicose Vein Disease is Related to Venous Hypertension. European Journal of Vascular and Endovascular Surgery. 2011 04;41(4):533-539. https://doi.org/10.1016/i.ejvs.2010.11.033

22. Evaluation of the smooth muscle cell component and apoptosis in the varicose vein wall. Histology and Histopathology. 200007 01;(33):745-752. https://doi.org/10.14670/HH-15.745

23. Ducasse E, Giannakakis K, Chevalier J, Dasnoy D, Puppinck P, Speziale F, Fiorani P, Faraggiana T. Dysregulated Apoptosis in Primary Varicose Veins. European Journal of

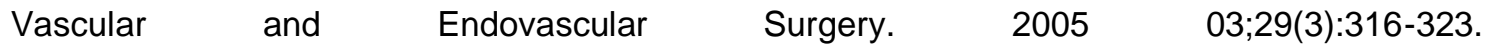
https://doi.org/10.1016/j.ejvs.2004.12.012

24. Harley B, Leung J, Silva E, Gibsonl L. Mechanical characterization of collagenglycosaminoglycan scaffolds. Acta Biomaterialia. 2007 07;3(4):463-474. https://doi.org/10.1016/j.actbio.2006.12.009

25. Wali M, Dewan M, Eid R. Histopathological changes in the wall of varicose veins. International Angiology. 2003;22(2):188-193.

26. Psaila JV, Melhuish J. Viscoelastic properties and collagen content of the long saphenous vein in normal and varicose veins. British Journal of Surgery. 1989 01;76(1):37-40. https://doi.org/10.1002/bjs.1800760112

27. Travers J, Brookes C, Evans J, Baker D, Kent C, Makin G, Mayhew T. Assessment of wall structure and composition of varicose veins with reference to collagen, elastin and smooth muscle content. European Journal of Vascular and Endovascular Surgery. 1996 02;11(2):230237. https://doi.org/10.1016/s1078-5884(96)80058-x 
INSUFICIENCIA VENOSA CRÓNICA Y LOS CAMBIOS ESTRUCTURALES EN LAS PAREDES DE LAS

VENAS - Dr. Luis Osvaldo Faringthon Reyes / Dr. Ouel Alvelis Sosa Veras

doi : https://doi.org/10.31434/rms.v4i2.172

28. Naim M, Elsharawy M. Histological assessment of the long saphenous vein in normal and varicosa veins. Egyptian Journal of Histology. 2005;28(2):281-290.

29. Martin SS, Alaminos M, Zorn TMT, Sánchez-Quevedo MC, Garzón I, Rodriguez IA, Campos A. The effects of fibrin and fibrin-agarose on the extracellular matrix profile of bioengineered oral mucosa. Journal of Tissue Engineering and Regenerative Medicine. 2011 Nov 03;7(1):10-19. https://doi.org/10.1002/term.490

30. Wali MA, Eid RA. Intimal Changes in Varicose Veins: An Ultrastructural Study.. Journal of Smooth Muscle Research. 2002;38(3):63-74. https://doi.org/10.1540/ismr.38.63

31. Khan AA, Eid RA, Hamdi A. Structural changes in the tunica intima of varicose veins: a histopathological and ultrastructural study. Pathology. 2000;32(4):253-257. https://doi.org/10.1080/pat.32.4.253.257

32. Wali MA, Eid RA. Smooth Muscle Changes in Varicose Veins: An Ultrastructural Study. Journal of Smooth Muscle Research. 2001;37(5,6):123-135. https://doi.org/10.1540/jsmr.37.123

33. Brunner F, Hoffmann C, Schuller-Petrovic S. Responsiveness of human varicose saphenous veins to vasoactive agents. British Journal of Clinical Pharmacology. 200807 07;51(3):219-224. https://doi.org/10.1046/j.1365-2125.2001.00334.x

34. Sansilvestri-Morel P, Rupin A, Badier-Commander C, Kern P, Fabiani J, Verbeuren TJ, Vanhoutte PM. Imbalance in the Synthesis of Collagen Type I and Collagen Type III in Smooth Muscle Cells Derived from Human Varicose Veins. Journal of Vascular Research. 2001;38(6):560-568. https://doi.org/10.1159/000051092

35. Wali M, Dewan M, Eid R. Histopathological changes in the wall of varicose veins. International Angiology. 2003;22(2):188-193.

36. Gandhi RH, Irizarry E, Nackman GB, Halpern VJ, Mulcare RJ, Tilson M. Analysis of the connective tissue matrix and proteolytic activity of primary varicose veins. Journal of Vascular Surgery. 1993 Nov;18(5):814-820. https://doi.org/10.1016/0741-5214(93)90336-k

37. Krasiński Z, Biskupski P, Dzieciuchowicz Ł, Kaczmarek E, Krasińska B, Staniszewski R, Pawlaczyk K, Stanisić M, Majewski P, Majewski W. The Influence of Elastic Components of the Venous Wall on the Biomechanical Properties of Different Veins Used for Arterial Reconstruction. European Journal of Vascular and Endovascular Surgery. 2010 08;40(2):224229. https://doi.org/10.1016/j.ejvs.2010.04.008

38. Sokolis DP. Experimental investigation and constitutive modeling of the 3D histomechanical properties of vein tissue. Biomechanics and Modeling in Mechanobiology. 201206 16;12(3):431-451. https://doi.org/10.1007/s10237-012-0410-y 
INSUFICIENCIA VENOSA CRÓNICA Y LOS CAMBIOS ESTRUCTURALES EN LAS PAREDES DE LAS

VENAS - Dr. Luis Osvaldo Faringthon Reyes / Dr. Ouel Alvelis Sosa Veras

doi : https://doi.org/10.31434/rms.v4i2.172

39. Kirsch $D$, Wahl W, Böttger $T$, Junginger $T$. Primary varicose veins-changes in the venous wall and elastic behavior. Der Chirurg. 2000;71(5):305-306.

40. Hernández Rivero M, Llanes Barrios J, Quiñones Castro M. Caracterización de la insuficiencia venosa crónica en consultas del Instituto de Angiología y Cirugía Vascular. Revista Cubana de Angiología y Cirugía Vascular. 2010;11(1):1-9.

41. Wilmanns C, Cooper A, Wockner L, Katsandris S, Glaser N, Meyer A, Bartsch O, Binder H, Walter PK, Zechner U. Morphology and Progression in Primary Varicose Vein Disorder Due to $677 \mathrm{C}>\mathrm{T}$ and 1298A $>\mathrm{C}$ Variants of MTHFR. EBioMedicine. 2015 02;2(2):158-164. https://doi.org/10.1016/i.ebiom.2015.01.006

42. Luebke T, Brunkwall J. Meta-analysis of subfascial endoscopic perforator vein surgery (SEPS) for chronic venous insufficiency. Phlebology: The Journal of Venous Disease. 2009 02;24(1):816. https://doi.org/10.1258/phleb.2008.008005

43. van Zuuren EJ, Fedorowicz Z, Pucci E, Jagannath VA, Robak EW. Percutaneous transluminal angioplasty for treatment of chron.

44. Martinez-Zapata MJ, Vernooij RW, Uriona Tuma SM, Stein AT, Moreno RM, Vargas E, Capellà D, Bonfill Cosp X. Phlebotonics for venous insufficiency. Cochrane Database of Systematic Reviews. 201604 06;. https://doi.org/10.1002/14651858.cd003229.pub3. 